\title{
Survival Benefit of Aspirin in Patients With Congestive Heart Failure: A Meta-Analysis
}

\author{
Sania Jiwani ${ }^{\mathrm{a}}$, Usman Mustafa ${ }^{\mathrm{a}}$, Sapna Desai ${ }^{\mathrm{b}}$, Paari Dominic ${ }^{\mathrm{a}, \mathrm{c}}$
}

\begin{abstract}
Background: There is no clear consensus on the use of aspirin (ASA) in patients with congestive heart failure $(\mathrm{CHF})$ due to its reported interaction with other cardio-prudent medications. The aim was to evaluate the effect of ASA on all-cause mortality and the frequency of hospitalization for heart failure in patients with CHF using metaanalysis, as well as to study the potential variables interacting with this effect.

Methods: Eligible studies were identified via a PubMed search, the "related article" feature and a manual search of references. Studies were included if they had a study population with CHF of any etiology, compared ASA to no ASA or placebo, and reported one or both of the following outcomes: 1) all-cause mortality and 2) the frequency of hospitalization for heart failure. Data were extracted and verified. We used the inverse variance method in a random-effects model to combine effect sizes.
\end{abstract}

Results: A total of 14 studies with a combined study population of 64,550 patients were included in the final analysis. All-cause mortality was found to be significantly lower in patients who were taking ASA $(P=0.003)$. When examining the use of ASA, no significant difference was found in the frequency of hospitalization for heart failure. ASA use was demonstrated to be more beneficial against mortality in studies with a larger percentage of patients on nitrates $(\mathrm{P}=0.008)$ and oral anticoagulants $(P=0.04)$. A significantly lower rate of hospitalization for heart failure was observed in patients who used oral anticoagulants and ASA concurrently $(\mathrm{P}=0.02)$.

Conclusions: ASA may have beneficial effects on mortality in patients with heart failure of all etiologies.

Keywords: Aspirin; Anti-platelet therapy; Congestive heart failure; Nitrates; Anticoagulation

Manuscript submitted November 2, 2020, accepted December 10, 2020

Published online January 12, 2021

aLouisiana State University Health Sciences Center - Center for Cardiovascular Diseases and Sciences, Shreveport, LA, USA

${ }^{b}$ Department of Cardiology, Ochsner Medical Center, New Orleans, LA, USA ${ }^{\mathrm{c} C}$ Corresponding Author: Paari Dominic, Department of Cardiology-Electrophysiology, Louisiana State University Health Sciences Center, 1541 Kings Highway, Shreveport, LA 71103, USA. Email: pdomi2@1suhsc.edu

doi: https://doi.org/10.14740/jocmr4389

\section{Introduction}

There is no clear consensus on the use of aspirin (ASA) in patients with congestive heart failure (CHF). ASA use is beneficial and is recommended in many conditions that frequently coexist with CHF, such as ischemic heart disease (IHD), atrial fibrillation (Afib) and diabetes mellitus (DM). The American College of Cardiology/American Heart Association (ACC/ AHA) and European Society of Cardiology (ESC) guidelines are equivocal regarding the use of ASA in CHF, especially in the absence of coronary artery disease (CAD). Various studies have reported conflicting results regarding the benefits and risks of ASA in this setting.

The current hypothesis behind the adverse effect of ASA in patients with CHF is its interaction with other cardio-prudent medications, particularly angiotensin converting enzyme inhibitors (ACEis). ASA antagonizes the beneficial vasodilator effects of ACEi; this has been well studied and reported on repeatedly. One mechanism of action of ACEi is an increase in prostaglandin synthesis via inhibiting breakdown of bradykinin by ACE. This pathway is thought to play an important role in counteracting the enhanced vasoconstriction in CHF [1]. ASA is known to reduce prostaglandin production via inhibition of cyclo-oxygenase. A systematic review of six randomized trials including 22,060 patients revealed weak evidence supporting the reduction in benefit from ACEi when used in combination with ASA [2]. Similarly, ASA was shown to result in a $30 \%$ attenuation of the natriuresis induced by spironolactone in healthy male subjects [3]. Chin et al further explored this interaction between ASA and eplerenone, but found no significant effect on clinical outcomes [4]. ASA has also been shown to interact with beta-blockers (BBs). In hypertensive male subjects, ASA pre-treatment lowered their hypotensive effect but did not affect their negative ionotropic or chronotropic effects [5]. In addition, ASA may interfere with reverse remodeling in patients with CHF treated with BB [6]. The known side effects of ASA on blood pressure control, renal function, and sodium and water retention may be particularly relevant in patients with CHF. When compared to clopidogrel, ASA has been shown to cause significant elevation of B-type natriuretic peptide (BNP) in patients with ischemic heart failure as well as in patients with CHF on ACEi [7]. Another aspect to consider is the potential contribution of ASA to the development of iron deficiency and anemia in these patients. The prevalence of iron deficiency in individuals with CHF has been reported to be as high as 30\%. Anemia and iron deficiency in CHF have been associated with 


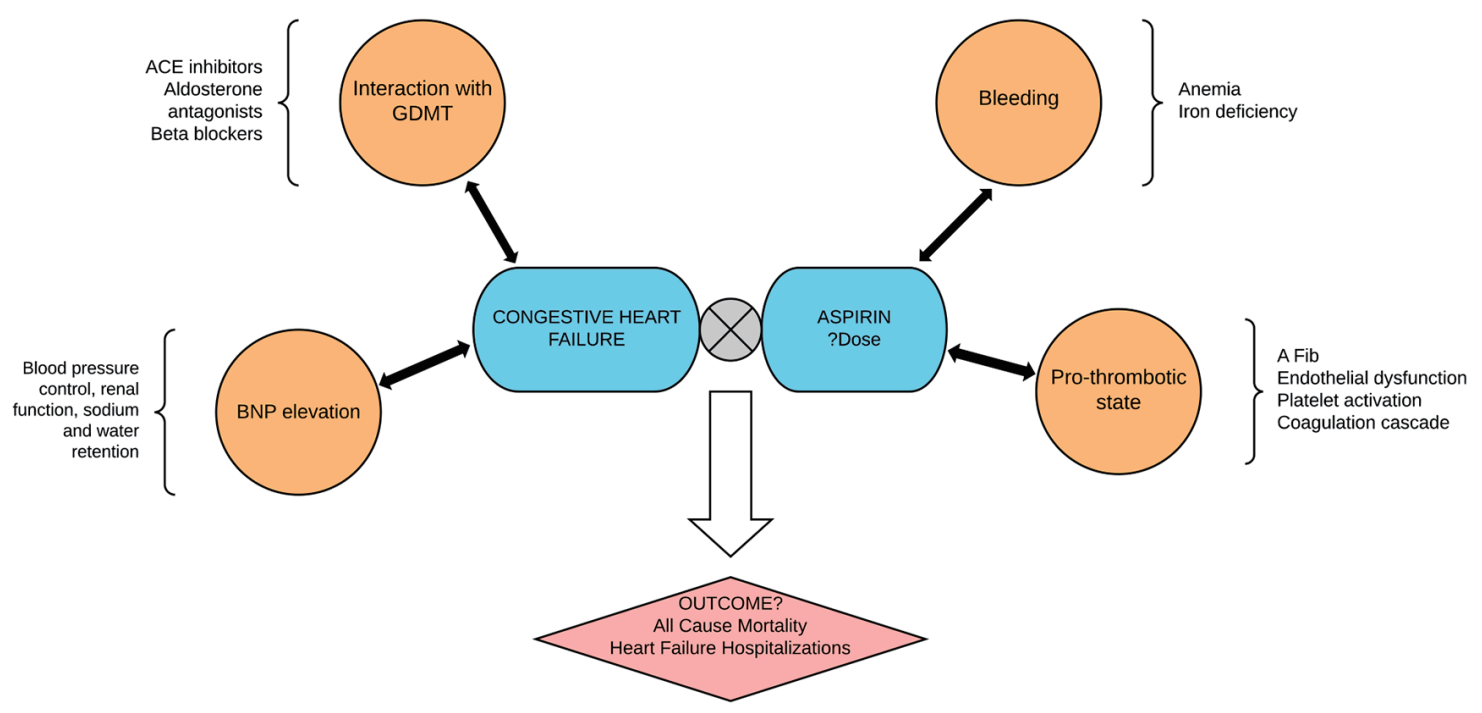

Figure 1. The use of aspirin in heart failure is complicated by the interaction of aspirin with goal-directed medical therapy in heart failure and the multiple pathophysiologic mechanisms in heart failure. The hard outcomes of aspirin use in patients with heart failure therefore have to do with the balance of such interaction and the homeostasis between bleeding and anti-thrombotic effects of aspirin. This study summarizes the mortality and heart failure outcomes with aspirin use in heart failure patients.

a higher all-cause and cardiovascular mortality [8].

The above described factors have the potential to result in worsening symptoms, increased hospitalizations and a higher mortality in patients with CHF. However, none of these theoretical interactions or adverse effects have been definitively proven to translate into poorer clinical outcomes resulting from the use of ASA (Fig. 1).

\section{Objectives}

The objective of this study was to evaluate the effect of ASA on all-cause mortality and the frequency of hospitalization for heart failure (HF) in patients with CHF, as well as to study the potential variables interacting with this effect using metaanalysis and meta-regression techniques, respectively.

\section{Materials and Methods}

We conducted this meta-analysis according to the recommendations of the Meta-analysis of Observational Studies in the Epidemiology Group (MOOSE).

\section{Search strategies}

PubMed was searched using the following keywords ((heart failure AND left ventricular dysfunction) AND aspirin) OR (("heart failure" [Mesh] OR cardiac failure OR heart decompensation OR myocardial failure) AND "ventricular dysfunction, left" [Mesh]) AND ("ASA" [Mesh] OR acetylsalicylic acid OR acid, acetylsalicylic OR 2-(acetyloxy)benzoic acid OR acylpyrin OR aloxiprimum OR colfarit OR dispril OR easprin OR ecotrin OR endosprin OR magnecyl OR micristin OR polopirin OR polopiryna OR solprin OR solupsan OR zorprin OR acetysal). A Google Scholar search was performed using the keywords "congestive heart failure", "ventricular dysfunction" AND "aspirin". The results were scanned to remove duplicate articles. We also used the "related article" feature on PubMed, along with a manual search of references, to identify additional studies. Only studies with full text available were included. We reviewed the full text of all relevant articles. English translations, if necessary, were obtained. Titles and abstracts were independently reviewed by two reviewers (SJ and UM) and cross-verified for inclusion. Details of the search strategy are reported in Figure 2.

\section{Inclusion and exclusion criteria}

Prospective or retrospective studies were included if they met the following inclusion criteria: 1) The study population included patients with a pre-existing diagnosis of CHF of any etiology. 2) The study compared ASA to no ASA or placebo. Studies that included two doses of ASA (low and high) were included as long as they were both compared against a third group that did not have ASA. When we encountered studies comparing groups of patients on ACEi with and without ASA to patients on ASA alone, we only included comparable groups, e.g., patients on ACEi with or without ASA. 3) The outcomes included one or both of the following: all-cause mortality and the rate of hospitalizations for HF. 4) We included studies irrespective of the dose of ASA used, co-morbidities and other medications.

\section{Data extraction}

Sania Jiwani extracted data from individual studies includ- 


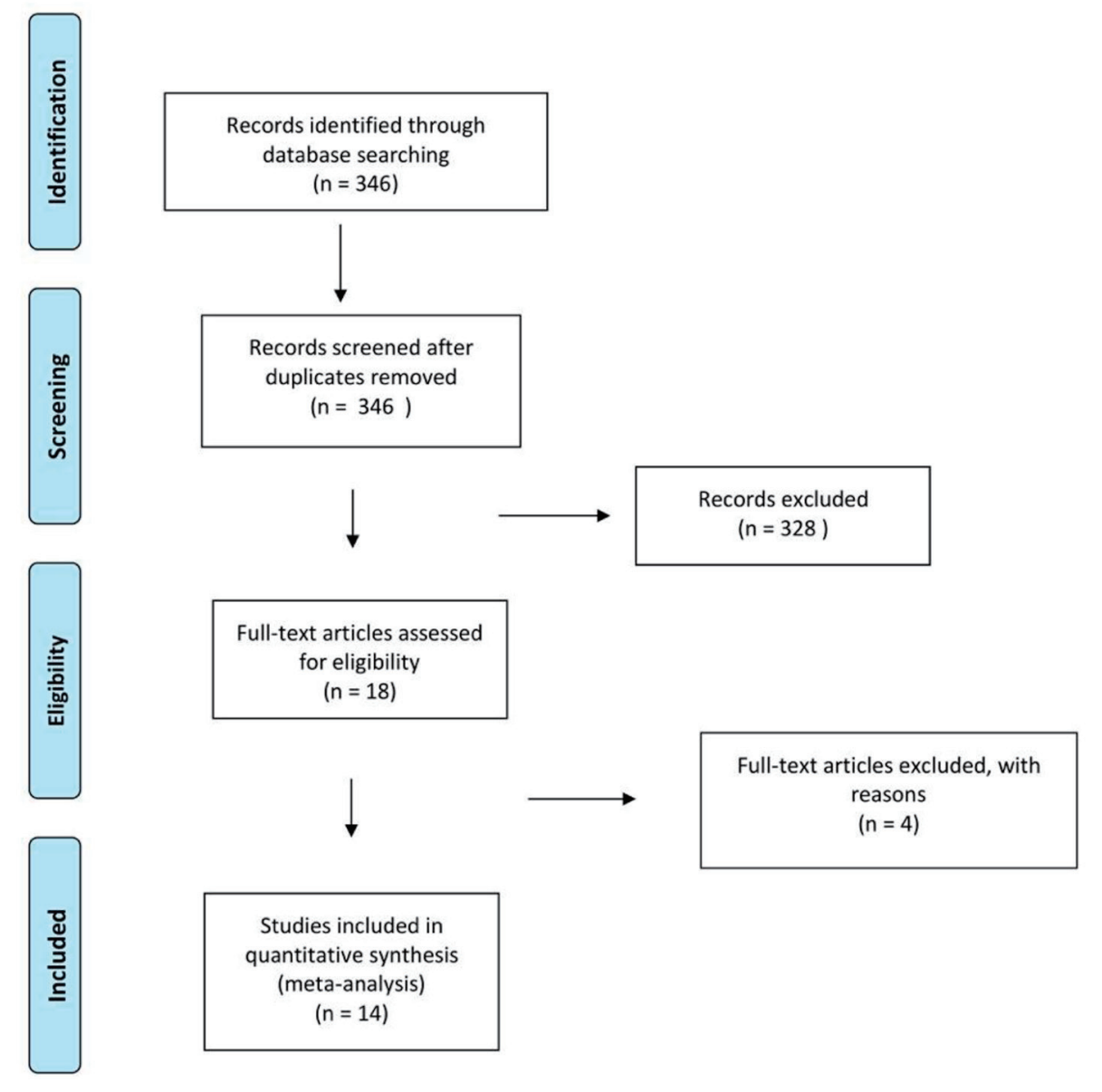

Figure 2. Preferred reporting items for systematic reviews and meta-analyses (PRISMA) flowchart of selection.

ing: 1) publication details, i.e., first author's last name, geographical region and year of publication; 2) study design; 3) characteristics of the study population including: age, sex, comorbidities (hypertension (HTN), DM, IHD, Afib) and medications (ACEi, BB, nitrate, oral anticoagulant (OAC)); and 4) adjusted hazard ratio (HR) with $95 \%$ confidence interval (CI). Event rates with sample size were extracted for the endpoints all-cause mortality and HF readmissions. Most studies used a Cox proportional hazards analysis or propensity score matching to calculate adjusted HR, which was considered the best estimate of the odds ratio (OR). Wherever reported, adjusted risks were used for the analysis. A second reviewer, Usman Mustafa, then verified all of the extracted data.

\section{Quality assessment}

The quality of each study and the risk of bias were evaluated using the Newcastle-Ottawa quality assessment scale for non-randomized studies. The following characteristics were assessed for sources of bias: 1) patient selection, including definitions of exposure and representation with respect to the larger population; 2) comparability of study groups and controlling for confounding factors by design or analysis; and 3) assessment and documentation of outcome, including duration and loss of follow-up. Studies were graded as "poor" if they met four or fewer of the nine criteria, "fair" if they met five to six criteria and "good" if they met more than six criteria. For uniformity, all studies, regardless of study design, underwent quality bias assessment using the retrospective cohort scoring tool.

\section{Statistical analysis}

We used Comprehensive Meta-Analysis version 3 (Biostat, Englewood, NJ) to perform all the analyses. OR with $95 \% \mathrm{CI}$ or adjusted HR was calculated using random effects model [9]. Statistical heterogeneity was determined using Q statistic and $\mathrm{I}^{2}$ index as proposed by Higgins and Thompson in their study [10]. An $\mathrm{I}^{2}$ of more than $56 \%$ or a $\mathrm{Q}$ test value at $5 \%$ was considered significant for heterogeneity [11]. A meta-regression analysis was performed when needed to investigate the factors contributing to heterogeneity. We adopted a weighted regression random effect model and carried out a univariate regression based on factors shown to affect mortality in HF and on availability of data for the majority of the studies included. A $\mathrm{P}$ value $<0.05$ was considered statistically significant for 
Table 1. Details of Studies Included in the Final Meta-Analysis

\begin{tabular}{|c|c|c|c|c|c|c|}
\hline First author, year & Region & $\begin{array}{l}\text { Study popula- } \\
\text { tion included }\end{array}$ & Study design & $\begin{array}{l}\text { Mean follow- } \\
\text { up (months) }\end{array}$ & $\begin{array}{l}\text { Outcome } \\
\text { reported }^{\text {a }}\end{array}$ & $\begin{array}{l}\text { Quality } \\
\text { assessment score }\end{array}$ \\
\hline Madelaire et al, 2018 [12] & Denmark & 7,680 & Retrospective cohort & 20.4 & 1,2 & 8 \\
\hline Bermingham et al, 2014 [13] & Ireland & 1,476 & Retrospective cohort & 31.2 & 1,2 & 8 \\
\hline Krumholz et al, 2001 [14] & USA & 1,110 & Retrospective cohort & 12 & 1,2 & 8 \\
\hline Cleland et al, 2004 [15] & UK, USA & 190 & Randomized control trial & 27 & 1 & 7 \\
\hline Harjai et al, 2001 [18] & USA & 378 & Observational & 13 & 2 & 8 \\
\hline Aumegeat et al, 2003 [19] & France & 693 & Retrospective cohort & 66.5 & 1 & 8 \\
\hline Chang et al, 2010 [20] & USA & 7,599 & Randomized control trial & 38 & 1,2 & 9 \\
\hline Chin et al, 2016 [4] & France & 2,737 & Randomized control trial & 21 & 1,2 & 7 \\
\hline Masoudi et al, 2005 [24] & USA & 24,012 & Retrospective cohort & 12 & 1,2 & 7 \\
\hline
\end{tabular}

aOutcome reported: 1: all-cause mortality; 2: hospitalization for heart failure.

all analyses. An Egger test with funnel plots of HR against standard error was used to evaluate for publication bias. We also performed a one-way sensitivity analysis of outcomes to estimate the effect of each study on all-cause mortality.

This is a meta-analysis of previously published studies. Hence, approval from the Institutional Review Board was not required.

\section{Results}

The keyword search yielded 346 studies. Additional studies were identified via manual cross-referencing of these studies. Application of the pre-specified inclusion criteria to all of these studies resulted in the inclusion of 14 studies in the final analysis [4, 12-24]. Details of the studies included are listed in Table 1. Six studies directly compared ASA to no ASA or placebo. Five studies compared ACEi with ASA to ACEi without ASA. Three studies had more than one ASA group: low dose and high dose. However, the high dose ASA group for one of these studies was not included as it was relatively small and not propensity matched.

Out of the total 14 studies, 10 were observational, one was a randomized control trial (RCT) and the remaining three were retrospective analyses of previous RCTs in which patients were not originally randomized based on the use of ASA.

Four studies were excluded from our study for the following reasons. 1) McAlister et al [25]: sizes of subgroups (patients on ASA with or without ACEi) not given and mortality outcome reported only for subgroups; cohort characteristics reported based on survival and HF readmission, not use of ASA. 2) De Boer et al [26]: cohort characteristics reported based on survival, not use of ASA. Neither the 3) WATCH trial [27] nor the 4) HELAS study [28] had placebo groups.

\section{All-cause mortality and HF hospitalizations with ASA use}

The combined study population included 64,550 patients. The baseline characteristics of these patients are reported in Supplementary Material 1 and 2 (www.jocmr.org). All-cause mortality was reported in 13 studies $(64,172$ patients) and found to be significantly lower in patients with CHF that were on ASA compared to those not on ASA (OR: $0.894,95 \%$ CI: 0.831 $0.962, \mathrm{P}=0.003, \mathrm{I}^{2}=66.70$; Table 2 and Fig. 3). Hospitalization for HF was reported in eight studies (56,270 patients). No significant difference was found in the frequency of hospitalization for $\mathrm{HF}$ in patients with $\mathrm{CHF}$ based on the use of ASA (OR: 0.91, 95\% CI: $0.798-1.037, \mathrm{P}=0.156, \mathrm{I}^{2}=83.48$; Table 2 and Supplementary Material 3, www.jocmr.org). Ex-

Table 2. Results of Meta-Analysis for Effect of Aspirin on All-Cause Mortality and Hospitalization for Heart Failure in Patients With $\mathrm{CHF}$

\begin{tabular}{lllll}
\hline Endpoint & Odds ratio & $\mathbf{9 5 \%}$ CI & P value & $\mathbf{I}^{\mathbf{2}}$ \\
\hline All-cause mortality & 0.894 & $0.831-0.962$ & 0.003 & 66.70 \\
Hospitalization for heart failure & 0.91 & $0.798-1.037$ & 0.156 & 83.48 \\
\hline
\end{tabular}

CHF: congestive heart failure; $\mathrm{Cl}$ : confidence interval. 


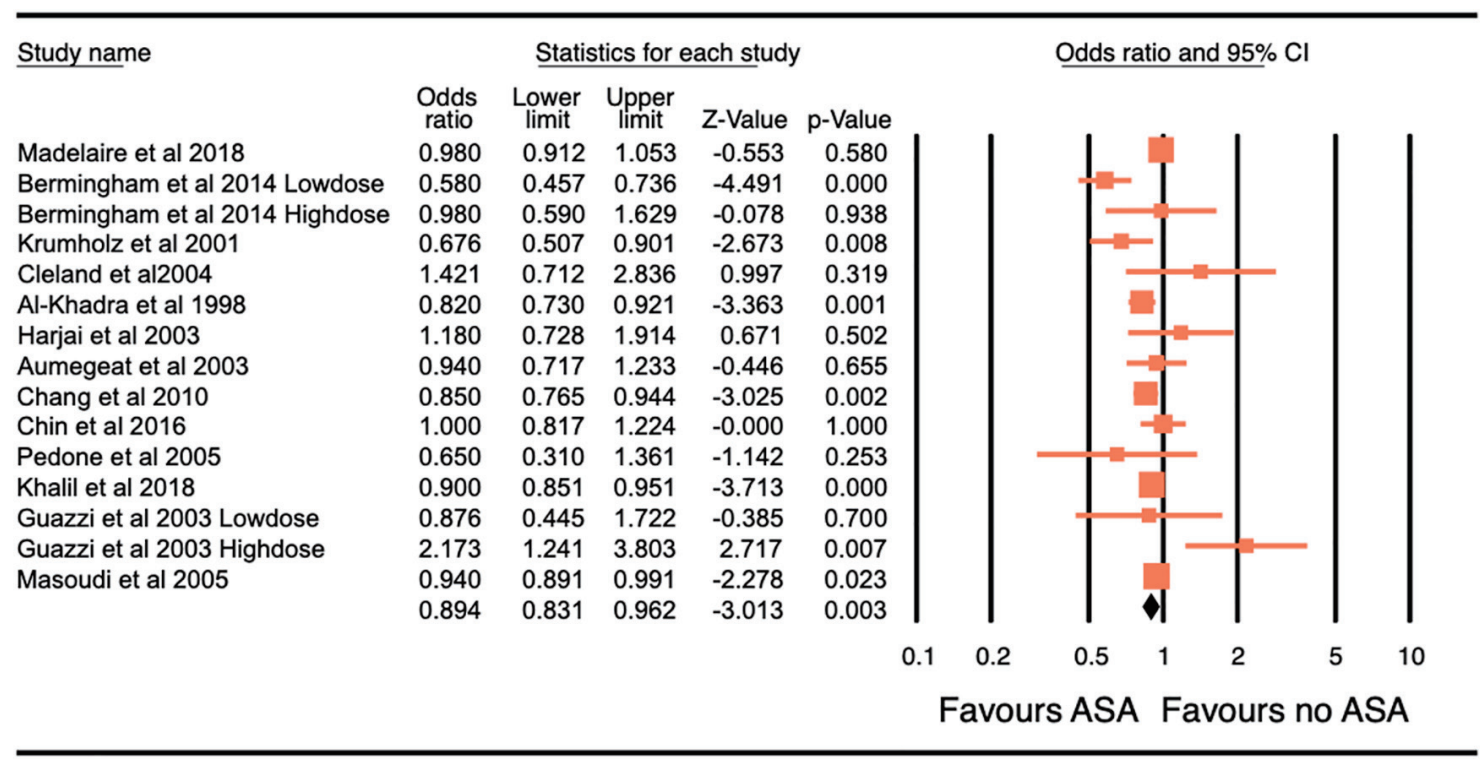

Figure 3. Forest plot of the odds ratio (OR) of all-cause mortality with the use of aspirin in patients with congestive heart failure. The overall significance of the results (OR: $0.894,95 \%$ confidence interval $(\mathrm{Cl}): 0.831-0.962, \mathrm{P}=0.003$ ), with the heterogeneity $\left(I^{2}=66.70 \%\right)$. The estimate of the OR of each study corresponds to the middle of the squares, and the horizontal line shows the $95 \% \mathrm{Cl}$. Pooling model using random (I-V heterogeneity) and the pooled OR estimated by weighting methods.

amination of the funnel plot for these studies did not reveal any publication bias (Supplementary Material 4 and 5, www.jocmr. org). In the sensitivity analysis, the omission of each study did not make a significant difference (Fig. 4), confirming the stability of our results.

\section{Outcomes of ASA in CHF patients - interaction of ASA with other HF medications}

The effect of ASA on the two endpoints in patients with $\mathrm{CHF}$ was further analyzed using meta-regression analyses for age, sex, HTN, DM, IHD, Afib, the concurrent use of ACEi, BB, nitrates and OAC. The results are summarized in Supplementary Material 6 and 7 (www.jocmr.org).

The studies did not report individual outcomes based on age, gender or race. Therefore, we used meta-regression analyses to study the interaction of these variables with the effect of ASA.

Information regarding age and gender of the study population was available in 12 and 14 studies respectively out of the total of 14 studies. Information regarding race of the study population was available in less than $50 \%$ of the included studies. Thus, meta-regression analysis for the variable race was not performed.

The variables of age, sex, HTN, DM, IHD, Afib, ACEi and $\mathrm{BB}$ had no significant interaction with the effect of ASA on mortality in these patients. However, in studies with a larger percentage of patients on nitrates and $\mathrm{OAC}$, it was observed that ASA conferred highly beneficial effects against mortality. This is shown in Figures 5 and 6.

The variables of age, sex, HTN, DM, IHD, Afib, ACEi, $\mathrm{BB}$ and nitrates had no significant association with the effect of ASA on hospitalization for HF in these patients. However, a significantly lower rate of hospitalization for HF was observed with the concurrent use of OAC and ASA. This is shown in Supplementary Material 8 (www.jocmr.org).

\section{Discussion}

The results of this meta-analyses show that ASA reduces allcause mortality in patients with CHF with no increase in the frequency of hospitalization for HF. Demographic factors and the presence of co-morbidities including HTN, DM and Afib did not affect the positive benefit of ASA in these patients. Surprisingly, this effect was not influenced by the percentage of patients with IHD in the studies, suggesting that this effect is not mediated through the known benefits of the drug in CAD. Any benefit of ASA in CHF of non-ischemic etiologies has not been reported in the literature.

\section{$\mathrm{HF}$ and thrombosis}

HF is now widely recognized to be a pro-thrombotic and inflammatory state, conferring an increased risk of stroke and thromboembolic events. A meta-analysis of 26 studies reported the incidence of ischemic stroke in individuals with $\mathrm{CHF}$ to be approximately 18 per 1,000 cases during the first year after diagnosis of CHF, and almost 50 per 1,000 cases of CHF by 5 years, which is significantly higher than that among the general population or in individuals with Afib on anticoagulation [29]. The extremely high prevalence of Afib with concomitant CHF may be a contributing factor to these observations [30]. Virchow's triad describes the three components that predispose thrombogenesis - abnormal blood flow, endothelial dysfunction 


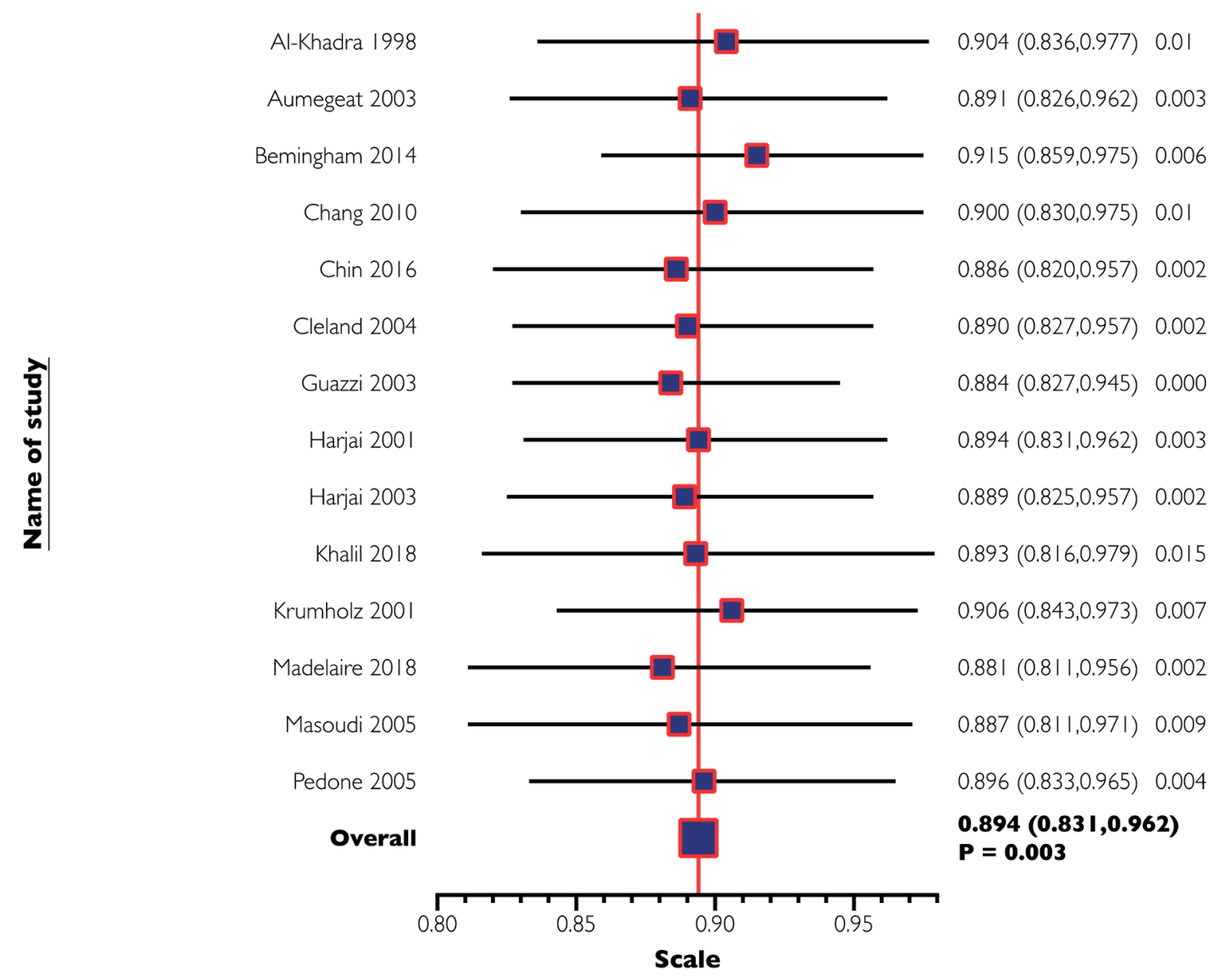

Figure 4. One-way sensitivity analysis of all-cause mortality. In this analysis, the omission of each study did not make a significant difference, confirming the stability of our results.

and a hypercoagulable state, all of which have been shown to play a role in CHF [31]. Several studies have demonstrated evidence of augmented and persistent activation of endothelium, platelets and the coagulation cascade in the setting of CHF [32, 33]. Compared to those in normal controls, levels of B-thromboglobulin, thrombin-antithrombin III complexes and D-dimer are increased in patients with $\mathrm{CHF}$, irrespective of its etiology. Circulating von Willebrand factor levels have been shown to be higher in individuals with $\mathrm{CHF}$, which also correlates with poorer clinical outcomes [32]. Patients with CHF have significantly higher levels of soluble P-selectin as well as expression of platelet-bound P-selectin compared to controls. However, the use of ASA did not appear to affect this disparity in P-selectin in this study [34]. These findings are suggestive of unexplained activation of the inflammatory and pro-thrombotic systems in patients with CHF. Therefore, it is not surprising that ASA with its anti-inflammatory and anti-thrombotic properties is beneficial in patents with $\mathrm{CHF}$, as demonstrated by our meta-analysis.

\section{ASA and goal-directed medical therapy in HF}

Based on conclusions drawn from data in prior studies, most of which were not founded on hard outcomes, ASA was thought to be harmful in patients with $\mathrm{CHF}$ due to its interaction with ACEi. However, our meta-regression analysis showed no statistically significant interaction with the use of ACEi or BB on the survival benefit from ASA in patients with CHF. An interesting result of our meta-regression analysis was that the survival benefit of ASA is higher in patients who concurrently use nitrates. The literature contains no previously known reported or studied interaction between ASA and nitrates. Further focused studies are needed to study this potential interaction. However, the effect of nitrates on platelets has been studied in the past. It was found that nitrates, via cGMP, inhibit the increase in cytosolic free calcium concentration in platelets induced by exposure to serotonin, vasopressin and plateletactivating factor [35]. This rise in the cytosolic free calcium is thought to be the principal mediator of platelet aggregation. In the context of augmented platelet activity in CHF as discussed above, this action may explain the synergistic effect of nitrates and ASA observed in our meta-analysis.

\section{ASA and oral anticoagulation in HF patients}

The reduction in mortality conferred by ASA in patients with $\mathrm{HF}$ was higher when the patients were also on OAC. Although 


\section{Regression of Log odds ratio on Nitrate}

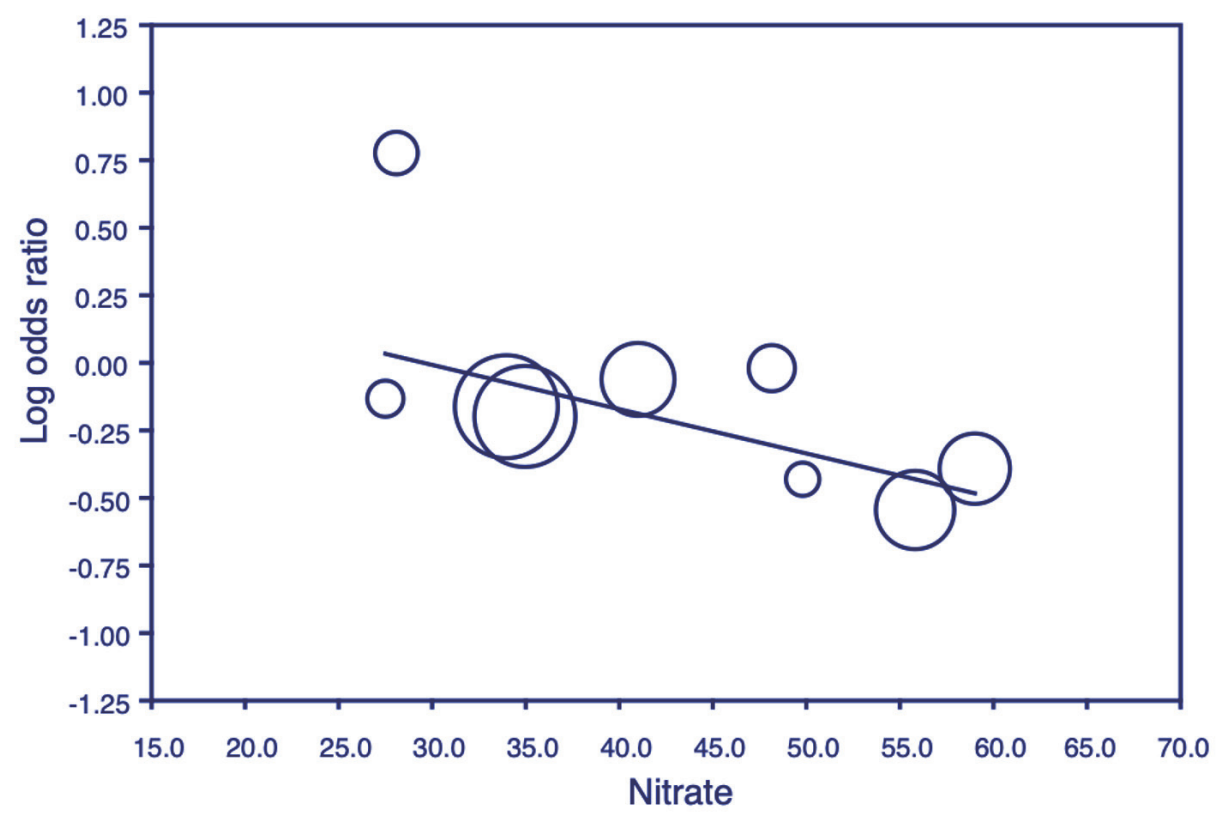

Figure 5. Meta-regression analysis for nitrates on all-cause mortality. Plot shows the log odds ratio (OR) of all-cause mortality against the use of nitrates in the studies. Each individual circle denotes the log of the OR (graphed on y-axis) of all-cause mortality based on the percentage of nitrate use in the study population (graphed on $\mathrm{x}$-axis). The size of the circle denotes the size of the total study population. The regression line denotes that the all-cause mortality decreases as the proportion of patients on nitrates increases.

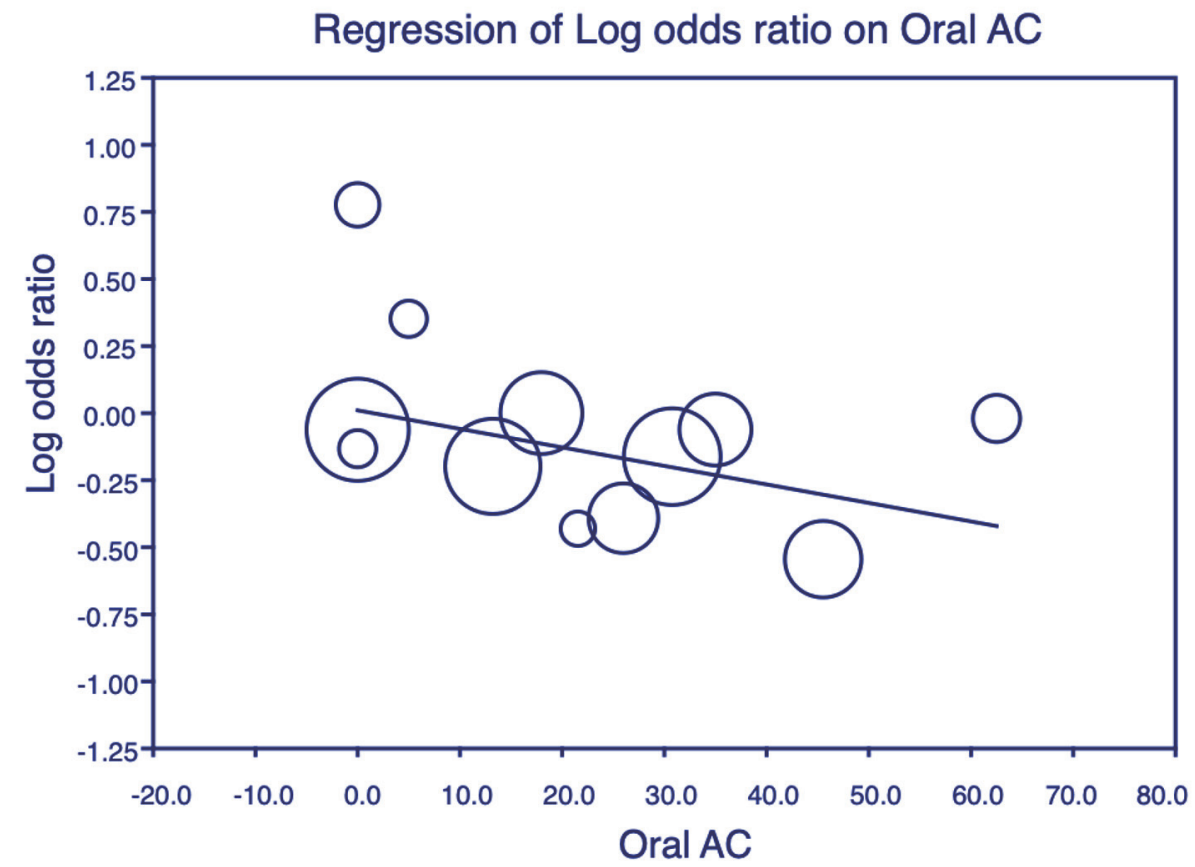

Figure 6. Meta-regression analysis for oral anticoagulant (OAC) on all-cause mortality. Plot shows the log odds ratio (OR) of all-cause mortality against the use of OAC in the studies. Each individual circle denotes the log of the OR (graphed on y-axis) of all-cause mortality based on the percentage of OAC use in the study population (graphed on x-axis). The size of the circle denotes the size of the total study population. The regression line denotes that the all-cause mortality decreases as the proportion of patients on OAC increases. 
ASA did not have a significant effect on hospitalization for HF in our meta-analysis, there was a significant relationship between ASA and OAC use in the included studies and reduced odds for hospitalization for HF. In view of the increased incidence of strokes and thromboembolic events in patients with $\mathrm{CHF}$, the use of OAC in CHF has been an area of interest and the focus of multiple studies. A meta-analysis including five trials and a total of 9,390 patients concluded a decreased risk of stroke and a higher risk of bleeding with the use of OAC, but no effect on mortality, hospitalizations or the risk of myocardial infarction [36]. While some researchers continue to monitor whether ASA may increase bleeding events when used in patients with HF, this was addressed in very few of the studies included. Madelaire et al [12] and Khalil et al [22] reported no difference in major bleeding events with the use of ASA. Cleland et al [8] found a higher incidence of minor hemorrhage in patients on ASA compared to those not taking ASA. The variation in definition and severity of bleeding events in these studies may explain the difference in results of the individual studies.

\section{Dose of ASA in HF}

The dose dependency of adverse effects of ASA in patients with CHF has been studied extensively. Glomerular filtration rate (GFR), sodium excretion and renal clearance are significantly lower with higher doses of ASA, with suggested clinical importance occurring not only at doses above $160 \mathrm{mg}$, but even at a dose of $80 \mathrm{mg}$ in patients with CHF [37]. The dose of ASA used was not reported in most of the studies in our metaanalysis, but where available, varied from 75 to $>300 \mathrm{mg} /$ day. The dose-dependent effect of ASA on the outcome of mortality was investigated in three of the studies included in the metaanalysis, albeit the dose classification was not consistent. Bermingham et al [13] found a reduced mortality risk in patients taking low-dose ASA (75 mg/day) compared to non-ASA users that was not seen in the cohort on high-dose ASA $(>75 \mathrm{mg} /$ day). Guazzi et al [23] reported that the combination of highdose ASA ( $\geq 325 \mathrm{mg}$ ) with an ACEi, but not low-dose ASA ( $\leq$ $160 \mathrm{mg}$ ), was independently associated with higher mortality. On the other hand, in a study by Khalil et al [22], no difference in outcomes was seen between low-dose ( $\leq 75 \mathrm{mg}$ /day) ASA and high-dose ASA (>75 mg/day), but low-dose ASA use conferred reduced mortality as compared to ASA non-users. However, in this study, the number of patients in the high-dose ASA cohort was relatively small and not propensity matched.

\section{Limitations}

This is a meta-analysis including observational studies and therefore carries the inherent limitations of the study design. All variables and outcomes were not reported in all the studies, resulting in a lower power. A small number of studies reported only rates for the outcomes measured and not adjusted HR. The dose of ASA used varied in the studies included in the meta-analysis and was not reported in all of them. Some of the studies tested ACEi with or without ASA, adding to the heterogeneity of studies. Although we found that ASA use was more beneficial against mortality in studies with a larger percentage of patients on nitrates, the specific nitrate drugs that were used in the studies could not be ascertained. Finally, some of the studies included patients from the 1990s when goal-directed medical therapy for CHF was not well defined and therefore BBs were under-utilized. Our meta-regression analysis did not show any interaction between ASA and BB on mortality and $\mathrm{HF}$ hospitalization outcomes in the trials. Moreover, despite this limitation, the findings offer an interesting finding that should be explored further with well-designed RCTs.

\section{Conclusions}

The use of ASA is safe in CHF and it does not interfere with the cardio-protective benefits of other medications as was feared in the past. ASA may have beneficial effects on mortality and possibly even the frequency of hospitalization for $\mathrm{HF}$ in patients with CHF of all etiologies. The benefits of ASA in $\mathrm{CHF}$ need to be explored further to provide evidence for more specific guidelines.

\section{Supplementary Material}

Suppl 1. Baseline Characteristics of Subjects in Individual Studies - Demographics and Comorbidities

Suppl 2. Baseline Characteristics of Subjects in Individual Studies - Medications

Suppl 3. Forest plot of the odds ratio (OR) of heart failure hospitalizations with the use of aspirin in patients with congestive heart failure. The overall significance of the results (OR 0.91, $95 \%$ CI $0.798-1.037, \mathrm{P}=0.156)$, with the heterogeneity $\left(\mathrm{I}^{2}\right.$ $=83.48 \%$ ). The estimate of the OR of each study corresponds to the middle of the squares, and the horizontal line shows the 95\% confidence interval (CI). Pooling model using Random (I-V heterogeneity) and the pooled OR estimated by weighting methods.

Suppl 4. Begg's funnel plot for the meta-analysis of all-cause mortality comparing aspirin and no aspirin in 13 studies. $\mathrm{P}$ value for the Begg test was 0.999. Log of odds ratio comparing all-cause mortality (vertical axis) is presented against the standard error (SE) of the log of OR (horizontal axis). The SE inversely corresponds to study size. Asymmetry of the plot can indicate publication bias.

Suppl 5. Begg's funnel plot for the meta-analysis of heart failure hospitalizations comparing aspirin and no aspirin in seven studies. P value for the Begg test was 0.999. Log of odds ratio comparing all-cause mortality (vertical axis) is presented against the standard error (SE) of the log of OR (horizontal axis). The SE inversely corresponds to study size. Asymmetry of the plot can indicate publication bias.

Suppl 6. Results of Meta-Regression Analyses for All-Cause Mortality

Suppl 7. Results of Meta-Regression Analyses for Heart Failure Hospitalizations

Suppl 8. Meta-regression analysis for OAC on heart failure 
hospitalizations. Plot shows the log odds ratio of heart failure hospitalizations against the use of OACs in the studies.

\section{Acknowledgments}

We thank Julia Esparza who assisted with the PubMed search for relevant studies. We thank Georgia Morgan for her assistance editing this manuscript.

\section{Financial Disclosure}

This publication was supported by an Institutional Development Award from the National Institutes of General Medical Sciences of the National Institutes of Health (NIH) under grant number P20GM121307 to C.G. Kevil.

\section{Conflict of Interest}

The authors declare no conflict of interest.

\section{Informed Consent}

Not applicable.

\section{Author Contributions}

Study concept and design: PD, SJ. Literature search: PD, SJ, UM. Acquisition, analysis and interpretation of data: PD, SJ, UM. Drafting of the manuscript: all authors. Critical revision of the manuscript for important intellectual content: PD, SD. Administrative, technical, or material support: PD. Study supervision: PD.

\section{Data Availability}

The authors declare that data supporting the findings of this study are available within the article.

\section{Abbreviations}

ASA: aspirin; CHF: congestive heart failure; IHD: ischemic heart disease; DM: diabetes mellitus; Afib: atrial fibrillation; CAD: coronary artery disease; ACEi: angiotensin converting enzyme inhibitor; BB: beta-blocker; OAC: oral anticoagulant; OR: odds ratio; HR: hazard ratio; $\mathrm{CI}$ : confidence interval

\section{References}

1. Dzau VJ, Packer M, Lilly LS, Swartz SL, Hollenberg NK, Williams GH. Prostaglandins in severe conges- tive heart failure. Relation to activation of the reninangiotensin system and hyponatremia. N Engl J Med. 1984;310(6):347-352.

2. Teo KK, Yusuf S, Pfeffer M, Torp-Pedersen C, Kober L, Hall A, Pogue J, et al. Effects of long-term treatment with angiotensin-converting-enzyme inhibitors in the presence or absence of aspirin: a systematic review. Lancet. 2002;360(9339):1037-1043.

3. Tweeddale MG, Ogilvie RI. Antagonism of spironolactone-induced natriuresis by aspirin in man. N Engl J Med. 1973;289(4):198-200.

4. Chin KL, Collier TJ, Pitt B, McMurray JJ, Swedberg K, van Veldhuisen DJ, Pocock SJ, et al. Aspirin does not reduce the clinical benefits of the mineralocorticoid receptor antagonist eplerenone in patients with systolic heart failure and mild symptoms: an analysis of the EMPHASIS-HF study. Eur J Heart Fail. 2016;18(9):1175-1181.

5. Sziegoleit W, Rausch J, Polak G, Gyorgy M, Dekov E, Bekes M. Influence of acetylsalicylic acid on acute circulatory effects of the beta-blocking agents pindolol and propranolol in humans. Int J Clin Pharmacol Ther Toxicol. 1982;20(9):423-430.

6. Lindenfeld J, Robertson AD, Lowes BD, Bristow MR, Investigators $\mathrm{M}$. Aspirin impairs reverse myocardial remodeling in patients with heart failure treated with betablockers. J Am Coll Cardiol. 2001;38(7):1950-1956.

7. Jug B, Sebestjen M, Sabovic M, Keber I. Clopidogrel is associated with a lesser increase in NT-proBNP when compared to aspirin in patients with ischemic heart failure. J Card Fail. 2006;12(6):446-451.

8. Cleland JG, Zhang J, Pellicori P, Dicken B, Dierckx R, Shoaib A, Wong K, et al. Prevalence and outcomes of anemia and hematinic deficiencies in patients with chronic heart failure. JAMA Cardiol. 2016;1(5):539-547.

9. DerSimonian R, Laird N. Meta-analysis in clinical trials. Control Clin Trials. 1986;7(3):177-188.

10. Higgins JP, Thompson SG. Quantifying heterogeneity in a meta-analysis. Stat Med. 2002;21(11):1539-1558.

11. Higgins JP, Thompson SG, Deeks JJ, Altman DG. Measuring inconsistency in meta-analyses. BMJ. 2003;327(7414):557-560.

12. Madelaire C, Gislason G, Kristensen SL, Fosbol EL, Bjerre J, D'Souza M, Gustafsson F, et al. Low-dose aspirin in heart failure not complicated by atrial fibrillation: a nationwide propensity-matched study. JACC Heart Fail. 2018;6(2):156-167.

13. Bermingham M, Shanahan MK, O'Connell E, Dawkins I, Miwa S, O'Hanlon R, Gilmer J, et al. Aspirin use in heart failure: is low-dose therapy associated with mortality and morbidity benefits in a large community population? Circ Heart Fail. 2014;7(2):243-250.

14. Krumholz HM, Chen YT, Radford MJ. Aspirin and the treatment of heart failure in the elderly. Arch Intern Med. 2001;161(4):577-582.

15. Cleland JG, Findlay I, Jafri S, Sutton G, Falk R, Bulpitt C, Prentice C, et al. The Warfarin/Aspirin Study in Heart failure (WASH): a randomized trial comparing antithrombotic strategies for patients with heart failure. Am Heart J. 2004;148(1):157-164. 
16. Al-Khadra AS, Salem DN, Rand WM, Udelson JE, Smith JJ, Konstam MA. Antiplatelet agents and survival: a cohort analysis from the Studies of Left Ventricular Dysfunction (SOLVD) trial. J Am Coll Cardiol. 1998;31(2):419-425.

17. Harjai KJ, Solis S, Prasad A, Loupe J. Use of aspirin in conjunction with angiotensin-converting enzyme inhibitors does not worsen long-term survival in heart failure. Int J Cardiol. 2003;88(2-3):207-214.

18. Harjai KJ, Nunez E, Turgut T, Newman J. Effect of combined aspirin and angiotensin-converting enzyme inhibitor therapy versus angiotensin-converting enzyme inhibitor therapy alone on readmission rates in heart failure. Am J Cardiol. 2001;87(4):483-487, A487.

19. Aumegeat V, Lamblin N, de Groote P, Mc Fadden EP, Millaire A, Bauters C, Lablanche JM. Aspirin does not adversely affect survival in patients with stable congestive heart failure treated with Angiotensin-converting enzyme inhibitors. Chest. 2003;124(4):1250-1258.

20. Chang SM, Granger CB, Johansson PA, Kosolcharoen P, McMurray JJ, Michelson EL, Murray DR, et al. Efficacy and safety of angiotensin receptor blockade are not modified by aspirin in patients with chronic heart failure: a cohort study from the Candesartan in Heart failure-Assessment of Reduction in Mortality and morbidity (CHARM) programme. Eur J Heart Fail. 2010;12(7):738-745.

21. Pedone C, Cecchi E, Matucci R, Pahor M, Carosella L, Bernabei R, Mugelli A, et al. Does aspirin attenuate the beneficial effect of ACE inhibitors in elderly people with heart failure? Drugs Aging. 2005;22(7):605-614.

22. Abi Khalil C, Omar OM, Al Suwaidi J, Taheri S. Aspirin use and cardiovascular outcome in patients with type 2 diabetes mellitus and heart failure: a population-based cohort study. J Am Heart Assoc. 2018;7(21):e010033.

23. Guazzi M, Brambilla R, Reina G, Tumminello G, Guazzi MD. Aspirin-angiotensin-converting enzyme inhibitor coadministration and mortality in patients with heart failure: a dose-related adverse effect of aspirin. Arch Intern Med. 2003;163(13):1574-1579.

24. Masoudi FA, Wolfe P, Havranek EP, Rathore SS, Foody JM, Krumholz HM. Aspirin use in older patients with heart failure and coronary artery disease: national prescription patterns and relationship with outcomes. J Am Coll Cardiol. 2005;46(6):955-962.

25. McAlister FA, Ghali WA, Gong Y, Fang J, Armstrong PW, $\mathrm{Tu}$ JV. Aspirin use and outcomes in a community-based cohort of 7352 patients discharged after first hospitalization for heart failure. Circulation. 2006;113(22):2572-2578.

26. de Boer RA, Hillege HL, Tjeerdsma G, Verheugt FW, van Veldhuisen DJ. Both antiplatelet and anticoagulant therapy may favorably affect outcome in patients with advanced heart failure. A retrospective analysis of the PRIME-II trial. Thromb Res. 2005;116(4):279-285.

27. Massie BM, Collins JF, Ammon SE, Armstrong PW, Cleland JG, Ezekowitz M, Jafri SM, et al. Randomized trial of warfarin, aspirin, and clopidogrel in patients with chronic heart failure: the Warfarin and Antiplatelet Therapy in Chronic Heart Failure (WATCH) trial. Circulation. 2009;119(12):1616-1624.

28. Cokkinos DV, Haralabopoulos GC, Kostis JB, Toutouzas $\mathrm{PK}$, investigators H. Efficacy of antithrombotic therapy in chronic heart failure: the HELAS study. Eur J Heart Fail. 2006;8(4):428-432.

29. Witt BJ, Gami AS, Ballman KV, Brown RD, Jr., Meverden RA, Jacobsen SJ, Roger VL. The incidence of ischemic stroke in chronic heart failure: a meta-analysis. J Card Fail. 2007;13(6):489-496.

30. Larned JM, Raja Laskar S. Atrial fibrillation and heart failure. Congest Heart Fail. 2009;15(1):24-30.

31. Sosin MD, Bhatia G, Davis RC, Lip GY. Congestive heart failure and Virchow's triad: a neglected association. Wien Med Wochenschr. 2003;153(19-20):411-416.

32. Chong AY, Freestone B, Patel J, Lim HS, Hughes E, Blann AD, Lip GY. Endothelial activation, dysfunction, and damage in congestive heart failure and the relation to brain natriuretic peptide and outcomes. Am J Cardiol. 2006;97(5):671-675.

33. Jafri SM, Ozawa T, Mammen E, Levine TB, Johnson C, Goldstein S. Platelet function, thrombin and fibrinolytic activity in patients with heart failure. Eur Heart J. 1993;14(2):205-212.

34. O'Connor CM, Gurbel PA, Serebruany VL. Usefulness of soluble and surface-bound P-selectin in detecting heightened platelet activity in patients with congestive heart failure. Am J Cardiol. 1999;83(9):1345-1349.

35. Negrescu EV, Sazonova LN, Baldenkov GN, Mukharliamov NM, Mazaev AV, Tkachuk VA. Relationship between the inhibition of receptor-induced increase in cytosolic free calcium concentration and the vasodilator effects of nitrates in patients with congestive heart failure. Int J Cardiol. 1990;26(2):175-184.

36. Sulaica EM, Macaulay TE, Helbing RR, Abo-Aly M, Abdel-Latif A, Wanat MA. A comparison of anticoagulation, antiplatelet, and placebo treatment for patients with heart failure reduced ejection fraction in sinus rhythm: a systematic review and meta-analysis. Heart Fail Rev. 2020;25(2):207-216.

37. Juhlin T, Jonsson BA, Hoglund P. Renal effects of aspirin are clearly dose-dependent and are of clinical importance from a dose of $160 \mathrm{mg}$. Eur J Heart Fail. 2008;10(9):892898. 